\title{
Energy Harvesting under Large Temperature Gradient - Comparison of Silicides, Half-Heusler Alloys and Ceramics
}

\begin{abstract}
This paper reports about a new four-step analysis method for thermoelectric (TE) materials. While the Seebeck coefficient is usually measured under small temperature gradient, we report here the measurement of TE materials under large temperature gradient. The examined materials were $\mathrm{Ag}$ - and $\mathrm{Bi}$-doped $\mathrm{Mg}_{2} \mathrm{Si}, \mathrm{Si}_{80} \mathrm{Ge}_{20}$, the ceramic composite $\mathrm{NaTaO}_{3}-\mathrm{Fe}_{2} \mathrm{O}_{3}$, the half-Heusler alloy (TiZr) NiSn, and the clathrates $\mathrm{Ba}_{8} \mathrm{Cu}_{x} \mathrm{Si}_{48-x}$ with $x=8,12$. The results show that the latter two metallic-like material groups have a low internal resistance. Hence, their output power is achieved at large electric currents with high carrier concentrations, while ceramic and silicides show the maximum output power at lower carrier concentration, which is considered to be a result of their large effective masses. The Arrhenius plot of the output energy yielded isosbestic-like points for ceramic-like materials, while the metal-like specimens show constant activation energies in the order of less than $0.1 \mathrm{eV}$. While all compared materials have reported $Z T$ values around 0.4, the output energy was similar and measured as around $0.01 \mathrm{~mW}$. Advantages of the metallic TE materials are their high carrier concentration and easy processing, disadvantages are their sensitivity to impurities and oxidation.
\end{abstract}

Keywords: measurement, thermoelectric, solid state physics

DOI 10.1515/ehs-2014-0013

\section{Introduction}

After the discovery of the thermoelectric (TE) material $\mathrm{Bi}_{2} \mathrm{Te}_{3}$ it has been utilized since the early 1960s for refrigeration (Goldschmid and Douglas 1954) and later for energy harvesting. Thermoelectric generators (TEGs) convert waste heat into electricity in the middle temperature

*Corresponding author: Wilfried Wunderlich, Materials Science Department, Faculty of Engineering, Tokai University, 259-1292 Hiratsuka, Japan, E-mail: wi-wunder@rocketmail.com range and are considered as a promising renewable energy source (Vining 1991; Bulusu and Walker 2008; Goupil et al. 2011; Feldhoff and Geppert 2014; Whitney 2014). The figure-of-merit $Z T$ is defined as

$$
\begin{gathered}
Z T=S^{2} \sigma T / \kappa \\
\text { with } S=U_{\text {See }} / \Delta T=U_{\text {See }} /\left(T_{\text {hot }}-T_{0}\right)
\end{gathered}
$$

where $S$ is the Seebeck coefficient, $\sigma$ the electric conductivity, $\kappa$ the thermal conductivity, $U_{\text {See }}$ the Seebeck voltage, $\Delta T$ the temperature gradient, $T_{\text {hot }}$ and $T_{0}$ the temperatures at the hot and cold sides of the specimen, respectively. TE materials can be roughly divided into two groups. Those with metallic-like behavior have large carrier concentration $n$ and hence large electric conductivity and small $S$. On the other hand, ceramic-like materials have low carrier concentrations with low electric conductivity and a large $S$. Hence, we approach the maximum of highest $Z T$ in TE development from the left side with ceramic-like materials and from the right side for metal-like materials, when drawing $Z T$ as a function of $n$.

The Seebeck coefficient is theoretically defined as small temperature gradient $\Delta T<10 \mathrm{~K}$, but in practical applications $\Delta T$ can be large (Wunderlich 2009; Min 2010) and recently theoretical explanation has made progress (Muto et al. 2009; De Marchi and Giaretto 2011; SandozRosado, Weinstein, and Stevens 2013). Examining this issue further is the objective of this paper. Recent studies (Goupil et al. 2011; Feldhoff and Geppert 2014) emphasized that the transformation of the heat flow into a flow of charged particles is combined with an increase in entropy. As the TE material is the power source, the achieved energy can be estimated by closing the electric circuit by load resistors $R_{\text {Load }}$ (Feldhoff and Geppert 2014; Wunderlich 2013a). An electric current $I$ is flowing according to Ohm's law and the electric output power $P_{\text {Out }}$ can be estimated as

$$
\begin{gathered}
I=U_{\text {See }} / R_{\text {Load }} \\
P_{\text {Out }}=U_{\text {See }}{ }^{*} I
\end{gathered}
$$


As in any electric circuit, the maximum current is achieved when $R_{\text {Load }}$ is minimal, and it is determined by the internal resistance of the power source. When increasing the resistance $R_{\text {Load }}$ in the closed circuit, a characteristic linear $I-U$ diagram is usually obtained, while deviations give valuable information of material properties. Circuit theory for the system in Figure 1 gives

$$
U / R_{\text {load }}=I=-U / R_{\text {internal }}+S \Delta T / R_{\text {internal }}
$$

where the left-hand side is for the load, the right-hand side is for the TE material, with $R_{\text {internal }}$ the internal resistance of the power source. The equality is due to current continuity according to Kirchhoff laws. Then simple algebra gives

$$
U=S \Delta T R_{\text {load }} /\left(R_{\text {load }}+R_{\text {internal }}\right)
$$
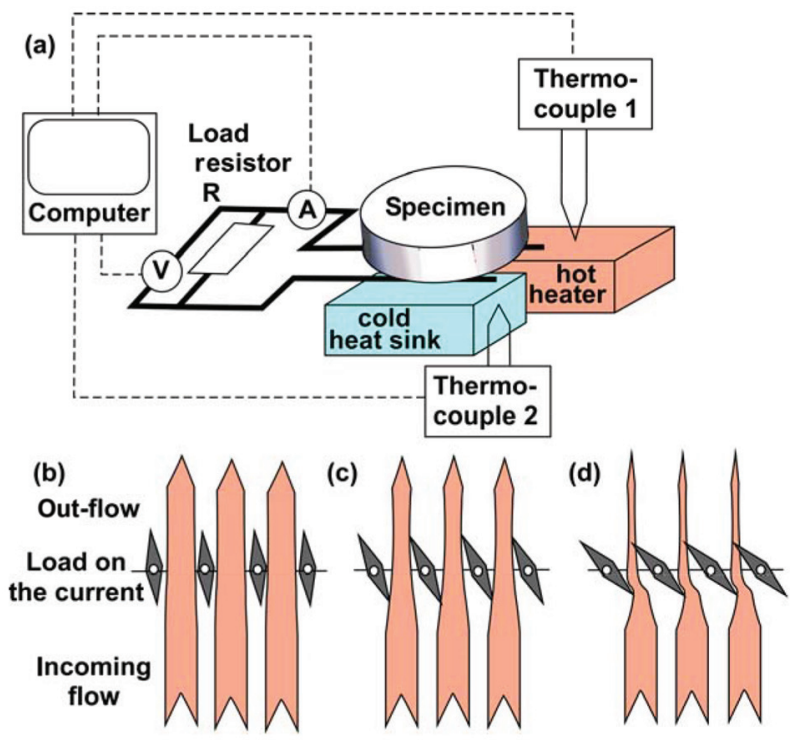

Figure 1: (a) Device for measuring of Seebeck voltage and current under applied load resistor under large temperature gradient, (b-d) Energy flow at a windmill as mechanical analogue for the conversion of an energy flow under resistance: (b) at low resistance energy flow goes through without transformation, (c) middle resistance means maximal transformation ratio, (d) while a large resistor is an obstacle for the energy flow. which means the power output is

$$
P_{\text {out }}=(S \Delta T)^{2} R_{\text {load }} /\left(R_{\text {load }}+R_{\text {internal }}\right)^{2}
$$

This function vanishes for both small $R_{\text {load }}$ and large $R_{\text {load}}$, but it has its maximum, when $R_{\text {load }}$ is equal to $R_{\text {internal }}$. With this method the internal resistance was estimated and displayed in Table 1.

This method has been suggested as an alternative to the above-mentioned measurement of $Z T$ and it is a challenge for theoreticians to evaluate the corresponding equations by using the Drude equation for electric conductivity $\sigma$ :

$$
\left.\sigma=e\left(n_{\mathrm{e}} * \mu_{\mathrm{e}}+n_{\mathrm{h}} * \mu_{\mathrm{h}}\right)\right)
$$

where $n_{\mathrm{e}}$ and $n_{\mathrm{h}}$ are electron and hole concentration and $\mu_{\mathrm{e}}$ and $\mu_{\mathrm{h}}$ are their mobilities, respectively. For heat-toelectricity conversion a large electron-phonon interaction (Wunderlich 2013a) is beneficial, while at the same time it decreases $\sigma$. Many papers attempt to understand and even calculate Seebeck coefficients, for example (Vining 1991; Yan et al. 2012), while predictions of new materials by calculations are still a challenge. For practical applications like in electronic devices thin films of TE are gaining interest (Wunderlich 2014c). In this paper we blend our experience on TE materials over the last decade with newly modified measurements using the load resistance method to examine the following materials.

TE materials are found within either of the groups of semiconductors, metals, or ceramics. Silicides have advantages over other TE materials that they are considered as environmental-friendly materials since its constituent elements are abundant in the earth's crust and nontoxic. The $\mathrm{Si}_{80} \mathrm{Ge}_{20}$ alloy (Vining 1990) is known for a long time and is already in use for many TEGs. With proper doping magnesium silicides can reach $Z T=1.4$ with $S=0.4 \mathrm{mV} / \mathrm{K}$, but their processing is difficult and they are sensitive to impurities (Liu et al. 2013; Gelbstein et al. 2014; Wunderlich et al. 2014b). They have a typical value of $Z T=0.4$. Another example of silicides are the promising $\mathrm{Ba}_{8} \mathrm{Cu}_{x} \mathrm{Si}_{48-x}$ clathrates with reported maximal $Z T=0.3$ (Yan et al. 2012),

\begin{tabular}{|c|c|c|c|c|c|c|c|c|}
\hline & NTF055 $=\underset{55 \% \mathrm{Ne}_{2} \mathrm{O}_{3}}{\mathrm{NaTaO}_{3}+}$ & $\mathrm{Si}_{80} \mathrm{Ge}_{20}$ & $\begin{array}{r}\mathrm{Mg}_{2} \mathrm{Si}+ \\
3 \% \mathrm{Bi}\end{array}$ & $\begin{array}{r}\mathbf{M g}_{2} \mathbf{S i}+ \\
3 \% \mathrm{Ag}\end{array}$ & $\mathrm{Ti}_{2} \mathrm{Zr}_{8} \mathrm{Ni}_{9} \mathrm{Sn}_{11}$ & $\mathrm{Ti}_{3} \mathrm{Zr}_{7} \mathrm{Ni}_{9} \mathrm{Sn}_{11}$ & $\mathrm{Ba}_{8} \mathrm{Cu}_{8} \mathrm{Si}_{28}$ & $\mathrm{Ba}_{8} \mathrm{Cu}_{12} \mathrm{Si}_{36}$ \\
\hline Max power $[\mu \mathrm{W}]$ & 12 & 8 & 8 & 3 & 12 & 14 & 14 & 3 \\
\hline Internal resistance $[\Omega]$ & 1,620 & 180 & 47 & 270 & 26 & 32 & 17 & 76 \\
\hline
\end{tabular}
while other clathrates can reach up to $Z T=1.4$ (Sootsman, Chung, and Kanatzidis 2009; Paschen et al. 2001). The

Table 1: Maximum power and internal resistance obtained by current theory analysis. 
doped half-Heusler alloy (Ti,Zr)NiSn can reach $Z T=0.8$ (Culp, Poon, and Tritt 2006; Muta et al. 2006; Wunderlich 2009) or even larger than $Z T=1.4$ (Sakurada and Sutoh 2005). Co-based ceramics have been reported as useful TE (Feldhoff and Geppert 2014; Wunderlich 2013a). More popular are perovskite such as $\mathrm{Nb}$-doped $\mathrm{SrTiO}_{3}$ (Ohta et al. 2005) as they show $Z T=0.4 . Z T=2.4$ has been reached at such interfaces (Ohta et al. 2007), but its realization in a device for applications is very challenging. The $\mathrm{NaTaO}_{3}-55$ $\mathrm{mol} \% \mathrm{Fe}_{2} \mathrm{O}_{3}$ composite, in the following called NTF055, shows a high electric current at the percolation composition (Wunderlich 2009). At such a composition interfaces between $\mathrm{p}$ - and $\mathrm{n}$-type materials show highest conductivity (Clerc et al. 1990).

In this paper we report a new experimental method for characterizing TE properties. After describing the experimental method, we describe the finding that the two classes of TEs can be clearly distinguished in these measurements. At least, we described another aim of this paper, the comparison of these material classes concerning their processing and practical handling, and describe their advantages.

\section{Experimental}

The specimens were prepared by weighing raw powders of $\mathrm{Mg}, \mathrm{Si}, \mathrm{Ba}, \mathrm{Cu}, \mathrm{Si}, \mathrm{Ti}, \mathrm{Ni}, \mathrm{Sn}, \mathrm{NaTO}_{3}$, or Fe (all high purity, $>99 \%$, Fine Chemical Ltd., Japan). Pellets $15 \mathrm{~mm}$ in diameter were cold pressed by a pressure of $80 \mathrm{MPa}$. The metallic (TiZr)NiSn, BaCuSi, and SiGe alloys were produced by conventional arc-melting (Wunderlich and Motoyama 2009). $\mathrm{Mg}_{2} \mathrm{Si}$ was produced by spark plasma sintering (SPS), for details see Wunderlich et al. (2014b). Ceramics were produced by conventional sintering at $1,000^{\circ} \mathrm{C}$ and $1,300^{\circ} \mathrm{C}$ in several steps. Experiences gained during these experiments are described in the discussion section. The home-made experimental measuring device as sketched in Figure 1(a) is used for characterization as described previously (Wunderlich 2009, 2013a) and a similar device is used by Feldhoff and Geppert (2014). In our device the specimen lies on one side on the heater, on the other side is a cold heat sink. Voltage, current, and temperatures $T_{0}$ and $T_{\text {hot }}$ are measured by electric multimeters (Sanwa PC510), recorded simultaneously in a computer and thereafter analyzed.

The idea of the newly introduced measurement procedure can be compared with the mechanical analogue of a windmill, which converts an air flow into rotational energy of rotor blades as shown in Figure 1(b)-(d). When the rotor blades are open as sketched in Figure 1(b), there is almost no resistance, but also the conversion ratio into mechanical energy is low. In Figure 1(c) the conversion ratio is highest; that mean output energy is highest. In Figure 1(d) the resistances of the tilted rotor blades are high, causing an obstacle for the wind flow rather than a smooth current. Similarly, the heat-into-electricity conversion at TE devices behaves like this mechanical model, when the electric circuit is closed with different resistor loads $(10 \Omega, 100 \Omega$, $1 \mathrm{k} \Omega, 100 \mathrm{k} \Omega, 1 \mathrm{M} \Omega$ ). When the electric resistor is small, the Seebeck voltage drops, while heat is almost completely converted into an electric current (Figure 1(d)), causing at the same time an obstacle to the incoming heat current. Middle size resistors result in highest power output as in Figure 1(c). A large electric resistor means almost no change in the heat flow, similarly as the unchanged wind flow in Figure 1(b). This phenomenon allows us to control the carrier concentration during measurement as described in the discussion section. When displaying the $I-U$ characteristics in the computer, almost all materials show straight lines according to their internal resistance. The lines were fitted and the output power was calculated according to eqs (3) and (4).

The time dependence scheme of the measurement is displayed in Figure 2. Typically, four different temperatures $T_{\text {hot }}$ (red line) during heating and one during cooling were adjusted by the heater, while cold temperature $T_{0}$ (light blue line) and $\Delta T$ (purple) are determined by the specimen's thermal conductivity and the environment. The Seebeck voltage was recorded all the times and shown as light green line in Figure 2. When heat flow was in equilibrium, the switch to the load resistance was closed and the occurring electric current (dark green

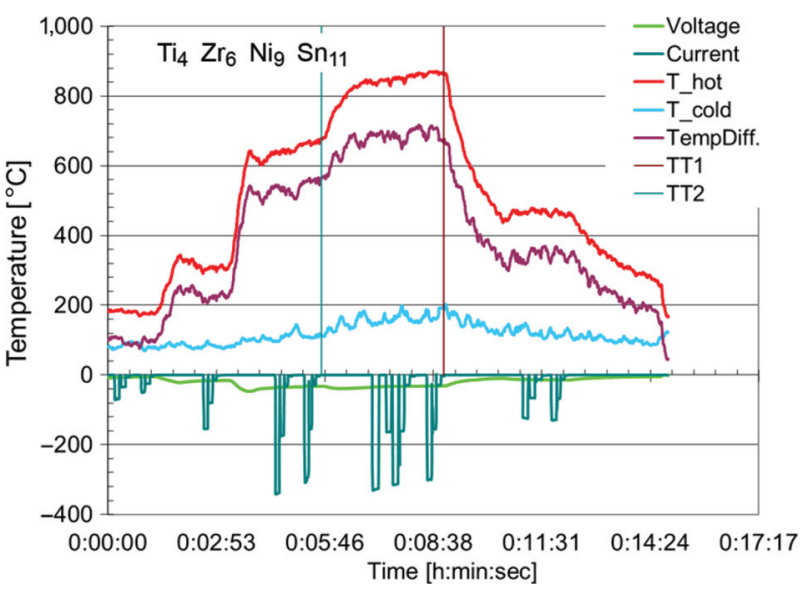

Figure 2: Typical measurement scheme for changing the hot temperature in five steps as displayed in red as a function of time, while temperature difference in purple, cold temperature in blue, Seebeck voltage in light green, and electric current in dark green is recorded. The vertical lines describe the transition points for color display in Figure 3. 

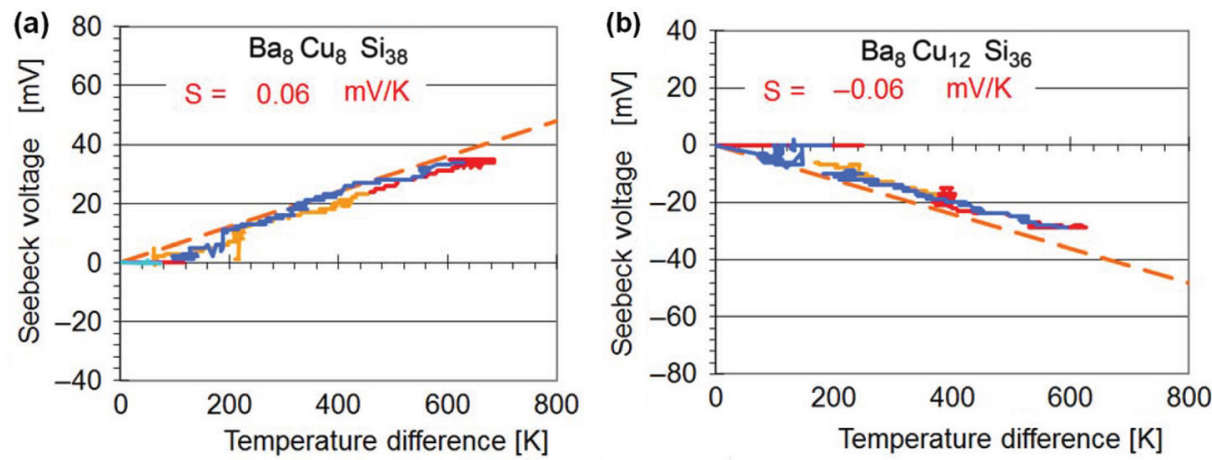

Figure 3: Seebeck voltages as a function of the temperature difference for (a) $\mathrm{Ba}_{8} \mathrm{Cu}_{8} \mathrm{Si}_{38}$, (b) $\mathrm{Ba}_{8} \mathrm{Cu}_{12} \mathrm{Si}_{36}$ specimens. The slope drawn in hatched line marks the Seebeck coefficient $S$. Orange color is used for heating up, red for constant temperature, and blue for cooling.

lines) recorded. Typical measurements of the Seebeck voltage are shown in Figure 3 without closing the circuit. In most cases straight lines were observed, which indicate the temperature-independent Seebeck coefficients. Some materials show deviations, which are discussed later in the following section.

The data were further processed by drawing the dependence on the load resistance as discussed later. Also the dependence on the five temperature steps was analyzed. The fit of the Arrhenius behavior was found by experience to be best, when a median temperature $T_{\mathrm{M}}$ was used as defined by the following equation:

$$
T_{\mathrm{M}}=T_{0}+1 / 2 \cdot \Delta T
$$

As the cold temperature $T_{0}$ was always maintained under the same conditions, $T_{\mathrm{M}}$ is proportional to the temperature difference $\Delta T$. The data of the output power as a function of reciprocal temperature were drawn in a double-logarithmic plot. They can be fitted with activated charge carriers $n_{\mathrm{A}}$, depending on an initial charge carrier concentration $n_{0}$ and the term with exaction energy $E_{A}$ and Boltzmann constant $k_{\mathrm{B}}$ according to the following equation:

$$
n_{A}=n_{0} \cdot \exp \left(\frac{E_{A}}{k_{\mathrm{B}} T}\right)
$$

This measuring procedure consisting of four steps was applied to eight specimens, as shown in the following section.

\section{Results and discussion}

As a first step, we measured the Seebeck voltage caused by the temperature difference. Such measurements have been published in previous papers for most of the specimens (Wunderlich 2009; Wunderlich and Motoyama 2009; Wunderlich 2013a; Wunderlich and Ohsato 2013b; Wunderlich et al. 2014b; Wunderlich 2014c). We show in Figure 3 new results for the (a) $\mathrm{Ba}_{8}$ $\mathrm{Cu}_{8} \mathrm{Si}_{38}$, (b) $\mathrm{Ba}_{8} \mathrm{Cu}_{12} \mathrm{Si}_{36}$ clathrates. The first one has p-type, the second n-type behavior. Even at a temperature difference of $\Delta T=700 \mathrm{~K}$ the Seebeck voltage does not reach $40 \mathrm{mV}$, leading to a Seebeck coefficient of +0.06 and $-0.06 \mathrm{mV} / \mathrm{K}$, respectively.

The next step is the measurement of the electric current, as shown in Figure 4 for all specimens, and as explained in the following. Figure 4(a) shows that the $\mathrm{NaTaO}_{3}-55 \mathrm{~mol} \% \mathrm{Fe}_{2} \mathrm{O}_{3}$ (NTF055) specimen reached $U_{\text {See }}=-300 \mathrm{mV}$ and $I=-180 \mu \mathrm{A}$ with $P=10 \mu \mathrm{W}$. When this specimen was produced several years ago and later published in Wunderlich and Motoyama (2009), it showed somewhat higher values, $U_{\text {See }}=-300 \mathrm{mV}$ and $I=-300 \mu \mathrm{A}$, and reached an output power of $20 \mu \mathrm{W}$ (Wunderlich and Ohsato 2013b). This outstanding material showed highest performance among all tested ones and reaches its maximum output power at the composition of the percolation. The reason for the slight degradation in current is apparently long-term oxidation; similarly Nb-doped $\mathrm{SrTiO}_{3}$ only shows high Seebeck coefficient, when produced under reduced atmosphere (Ohta et al. 2005). Also the measurement on the next specimen, the p-type $\mathrm{Si}_{80} \mathrm{Ge}_{20}$, is shown here for the first time in Figure 4(b). Next, Figure 4(c) shows the $\mathrm{Mg}_{2} \mathrm{Si}$ specimen doped with $3 \% \mathrm{Bi}$ (in the following called $\mathrm{Mg}_{2} \mathrm{Si}-\mathrm{Bi} \mathrm{K}$ ), which refers to SPS sintered from purchased $\mathrm{Mg}_{2} \mathrm{Si}$ powder (Wunderlich et al. 2014b), and (d) p-type $\mathrm{Mg}_{2} \mathrm{Si}$ doped with 3\% Ag (called $\mathrm{Mg}_{2} \mathrm{Si}-\mathrm{AgS}$, Wunderlich et al. 2014b). All measurements could confirm a straight line in the $I-U$ plot. The hatched line was fitted to the measured data points at maximum temperature difference (red line) and matched perfectly. Also the corresponding data points for 

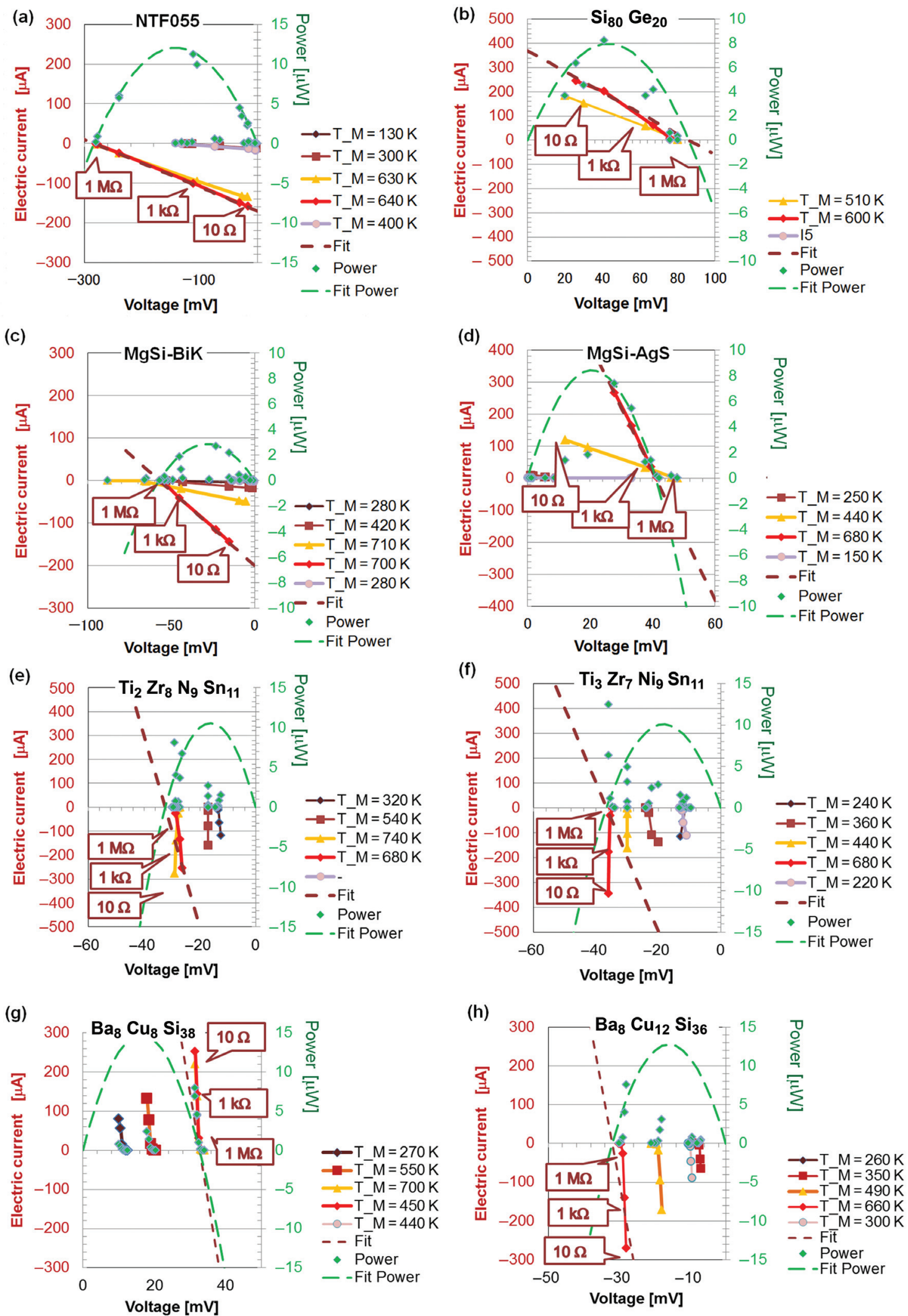

Figure 4: Electric current and output power as a function of Seebeck voltage for different temperature gradients and materials as marked. Three of the applied load resistors are marked with labels. 
the power match perfectly. Feldhoff and Geppert (2014) and Wunderlich (2013a) found this behavior for Co-based ceramics as well.

In contrary, the metallic-like half-Heusler and clathrate specimens show different behavior. As mentioned before (Figure 3) the maximum Seebeck voltages are much lower and are in the range of $30 \mathrm{mV}$, while the electric current reaches or even exceeds $I=-300 \mu \mathrm{A}$. In the $I-U$ plot the lines are almost vertical, indicating a very low internal resistance. Wunderlich, Amano, and Matsumura (2014a) reported similar behavior, when the dependence on the composition of (Ti,Zr)NiSn specimens was examined. The only exception was specimens with low Seebeck coefficients, especially $\mathrm{Ti}_{0.2} \mathrm{Zr}_{0.8} \mathrm{NiSn}$. In this case, the $I-U$ lines are tilted in a similar way as the abovementioned semiconductors indicating such a behavior. The envelope of the maximum current for all temperatures lies on a straight line through the origin. Apparently, there occurs a phenomenon which limits the maximum current. The fit was performed in such a way that a compromise between a middle value of the current and the power was achieved. As a consequence, the measured data points of the power lie on the side of the high absolute voltage values (left for $n$-type, right for $\mathrm{p}$-type).

The third step in the measurement analysis is shown in Figure 5. For each specimen the whole data set of the electric output power was drawn in a double-logarithmic plot against the load resistance. As mentioned in the introduction, a large resistor decreases the mobility of charge carriers $\mu_{\mathrm{e}}$ or $\mu_{\mathrm{h}}$. When mobility is reduced, the recombination of exited carriers is likely to occur. We can also express it in this way: When charge carriers are already present, the excitation of new ones is suppressed, because it is well known that semiconductor devices have best performance, when they contain a $\mathrm{p} / \mathrm{n}$ junction, in which the different types of carriers are accelerated and separated. Examples are devices such as light emitting diodes, solar cells, or photocatalyst materials, where this effect is known as antenna effect (Wang et al. 2006). Such a regime corresponds to the right side of the plot, while the left side indicates a regime of a large number of carriers. Many papers have emphasized such an optimal carrier concentration for maximum $Z T$, as a large $n_{\mathrm{e}}$ or $n_{\mathrm{h}}$ increases the electric conductivity, while a low $n_{\mathrm{e}}$ or $n_{\mathrm{h}}$ is responsible for a high Seebeck coefficient. Under these considerations the metallic specimens (Figure 5(e)-(h)) lie all in the regime of the high load resistors and show the power maximum at the lowest load resistance, which means highest carrier concentration.

The curves for low temperature are always lower in output power and parallel to those for high temperature.
$\mathrm{P}$ - and n-type TEs show equivalent behavior. The ceramic and semiconducting specimens, however, show a remarkable decrease in power in the high carrier regime. In other words, in the area where a potentially high power could be achieved, a certain phenomenon causes a drop in the output power. This phenomenon is apparently related to interactions between carriers, as it occurs in the high carrier regime. One explanation could be the large effective mass of ceramics caused by a cloud of polarization with a certain spatial diameter. Experience from interface observations showed that the interaction of electromagnetic waves from one material can exceed more than two unit cells (Wunderlich 2014c), and we assumed that the diameter of polarization is in the same order of magnitude. When the carrier concentration, which is actually a carrier density, becomes higher and higher, at a certain turning point there is simply not enough space left for the movement of carriers without interaction and hence, their large value of the effective mass breaks down. With increasing temperature the maximum of the output power shifts from the high resistance regime, which means low mobility and hence low carrier concentration per time unit, toward the regime of high carrier density. All curves seem to have the same shape, while small deviations are considered as lack of measurement accuracy. During cooling the reverse direction of the heat flow causes in some specimens a decrease in output power as $\Delta T$ is significantly lowered. As a conclusion of this section we can state that in such ceramic-like materials, which are based on large effective masses, an increase of charge carrier concentration by large electric currents is ineffective. The spatial extension of the polarization clouds is considered as a natural barrier which limits the effect of further increase in carrier flux.

The final step in our measurement analysis is the Arrhenius plot as shown in Figure 6. As we have measured the Seebeck voltage at five different temperature differences, we are able to plot the output power as a function of the inverse temperature $T_{\mathrm{M}}$. The metallic-like materials show parallel lines in Figure 6(e)-(h) with the same activation energies $0.1 \mathrm{eV}$ as displayed in red for the large carrier concentration regime and in blue for the low one. In this case the drop of output power with increasing load resistance is suggested to be caused by the Thomson effect (Min 2010). The Ti $i_{0.3} \mathrm{Zr}_{0.7} \mathrm{Ni}_{0.9} \mathrm{Sn}_{1.1}$ specimen, which has the highest output, shows the lowest activation energy of $0.06 \mathrm{eV}$. For ceramic-like materials $\mathrm{Mg}_{2} \mathrm{Si}$ such low activation energies of 0.08 are also found (Figure 6(c),(d)), but only in the regime of low carrier concentration. For the $\mathrm{NaTaO}_{3}-\mathrm{Fe}_{2} \mathrm{O}_{3}$ composite and SiGe they are higher (0.15 eV in Figure 6(a),(b)). 

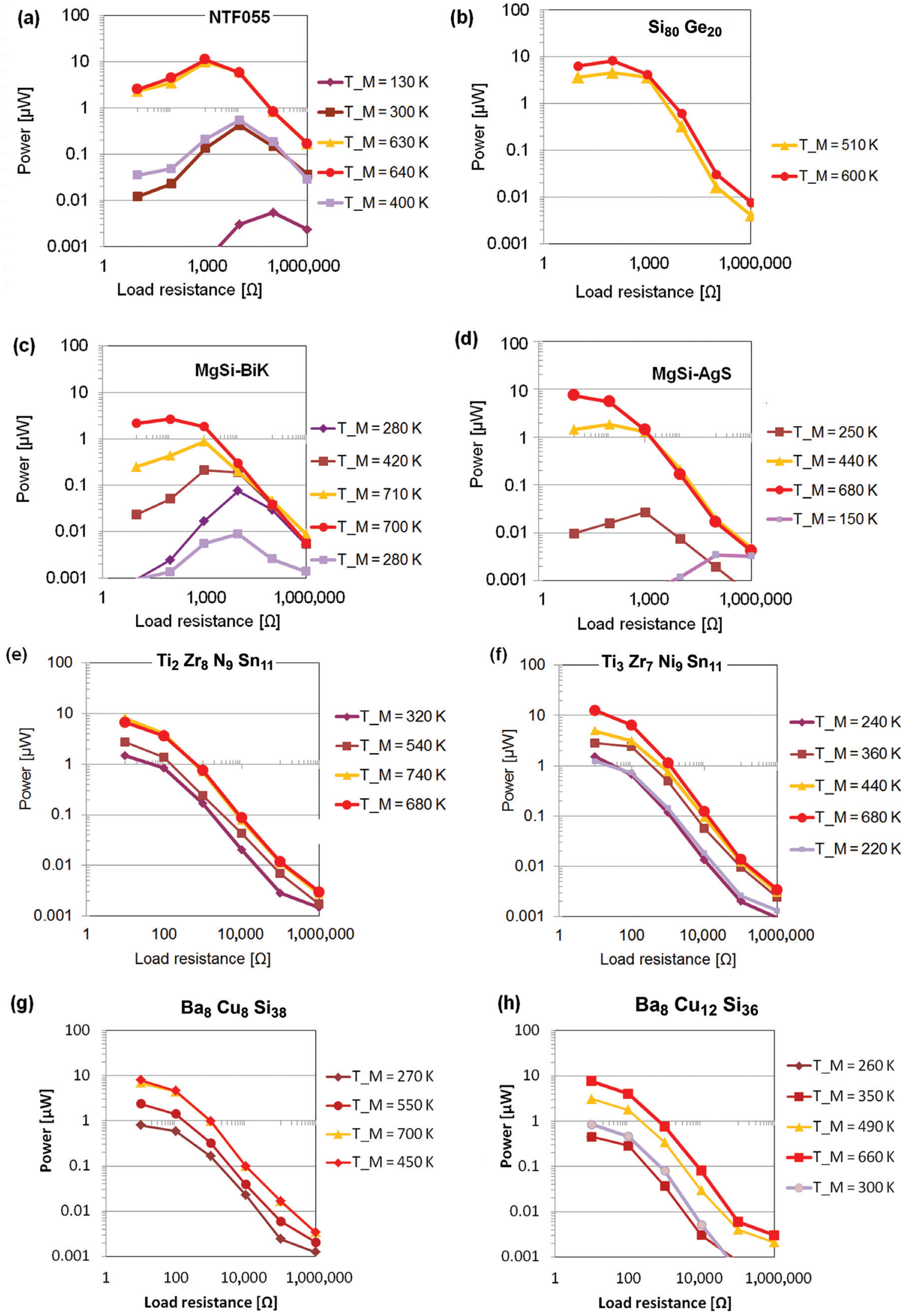

Figure 5: Electric output power as a function of load resistance for different temperature gradients and materials as marked. 


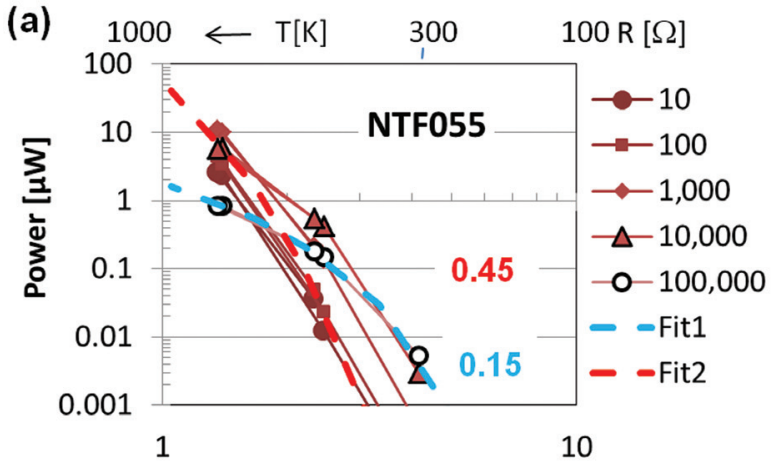

inverse Temperature 1000/T [1/K]
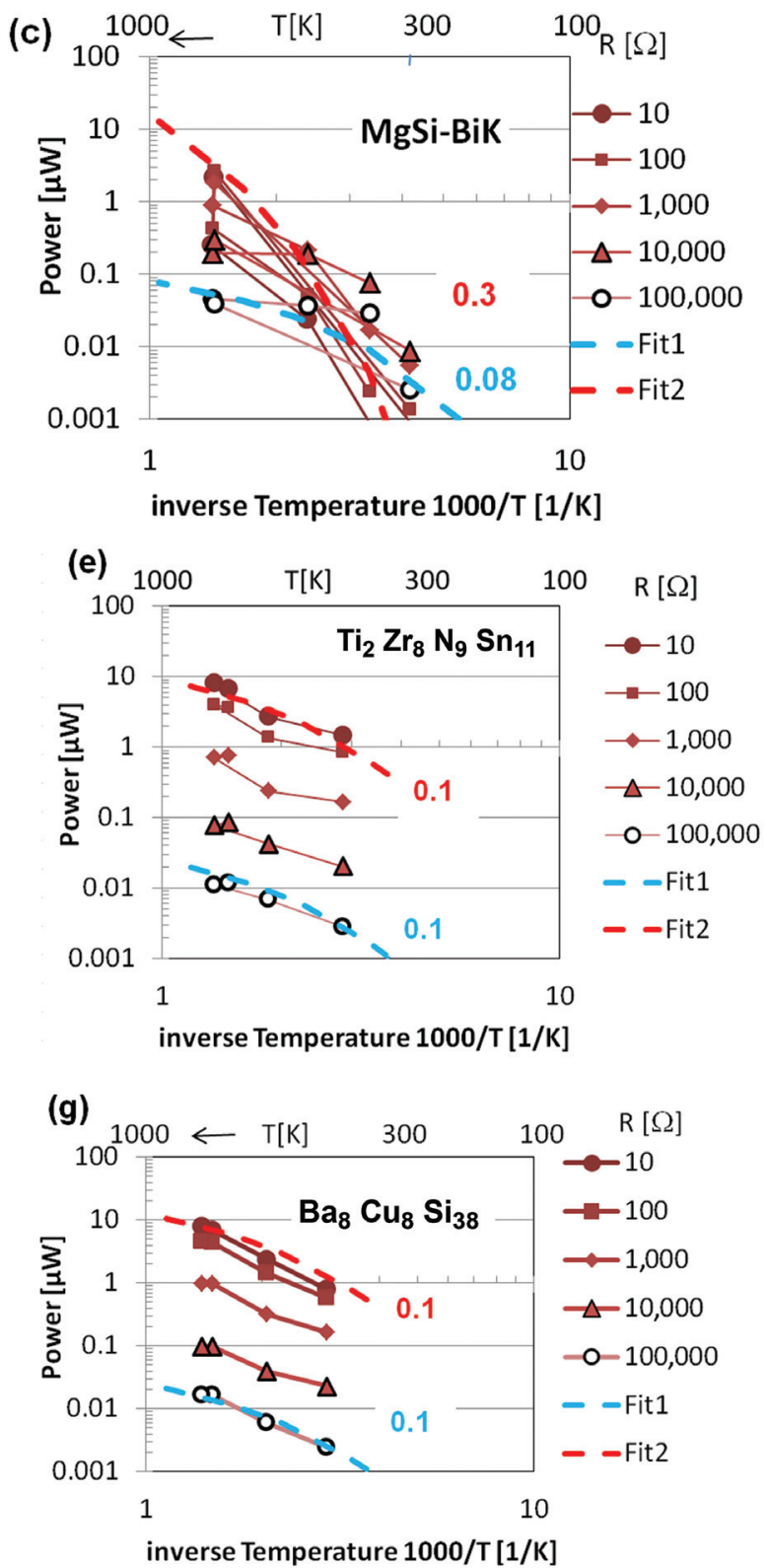
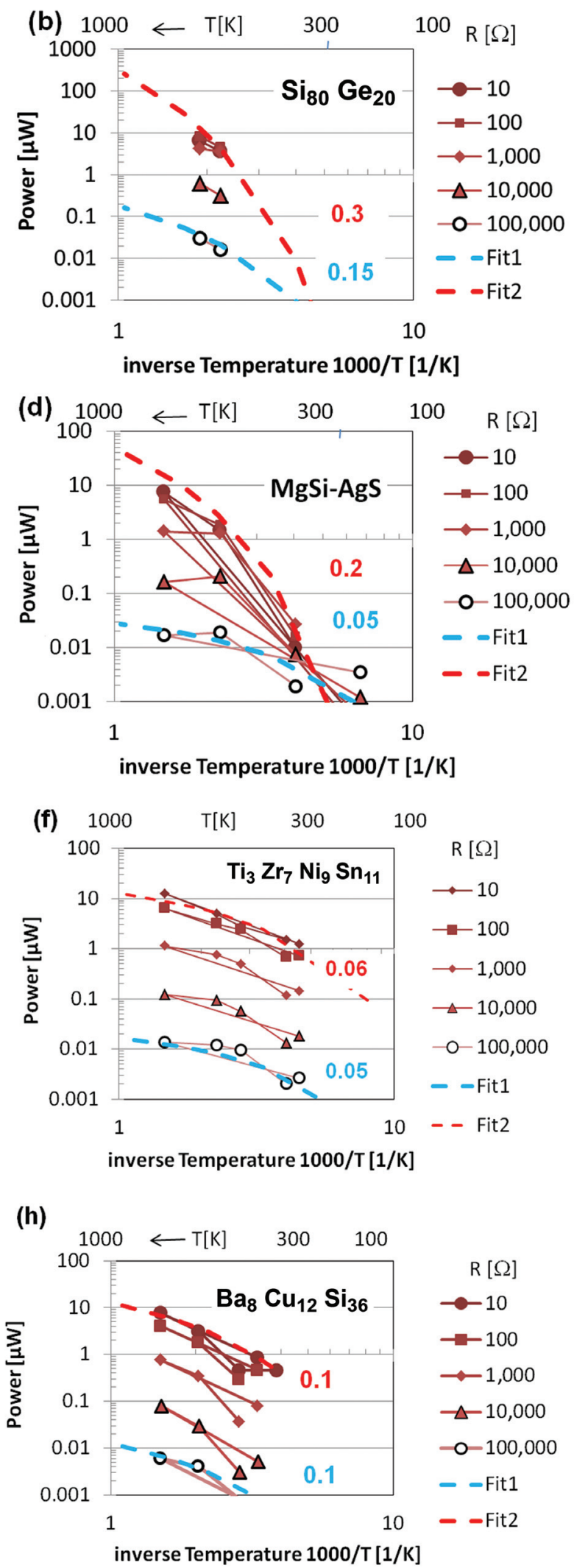

Figure 6: Electric output power as a function of the inverse temperature $T_{\mathrm{M}}$ for the materials as marked. The fitted activation energies in [eV] are given in red for the low load resistance of $10 \Omega$ and in blue for the high resistance of $1 \mathrm{M} \Omega$. 
The activation energies in the regime of high carrier concentration are significantly higher, ranging from 0.2 to $0.45 \mathrm{eV}$. We call the intersection point between both graphs isosbestic point according to a similar phenomenon in optical spectroscopy. For metallic-like specimens the isosbestic points would be far in the low-temperature region. The more the materials become ceramic-like in their character, the more the isosbestic point shifts to higher temperatures. At the same time the activation energy for the high carrier concentration regime increases. This analysis can explain why metallic- and ceramic-like TEs behave so different. For prediction of new materials we need to find correlations between these activation energies and the element properties.

The analysis according to circuit theory yields the values for maximum power and internal resistances as summarized in Table 1. It can be concluded that maximum output power depends not only on the Seebeck coefficient, but also strongly on the internal resistance. The metallic-like specimens based on TiNiSn and BaCuSi show the highest values of output power and the lowest resistance, while their Seebeck coefficient is only moderate. An additional information not shown in Table 1 is the fact that the internal resistance increases with temperature $T_{\mathrm{M}}$, in the same manner as expected from thermal vibrations.

We summarize now all findings from the viewpoint of experimental experience. Comparing all specimens, the maximum output power is around 8 to $10 \mu \mathrm{W}$, with the only exception being the Bi-doped $\mathrm{Mg}_{2} \mathrm{Si}$, because microstructure is not optimized or oxygen impurity level is too high. As mentioned in the introduction the literature reported for all of these specimens at figure-of-merit around $Z T=0.4$. The $\mathrm{Ti}_{3} \mathrm{Zr}_{7} \mathrm{Ni}_{9} \mathrm{Sn}_{11}$ specimen has the highest output power of $12 \mu \mathrm{W}$. The $\mathrm{NaTaO}_{3}-55 \mathrm{~mol} \%$ $\mathrm{Fe}_{2} \mathrm{O}_{3}$ composite showed the highest value of $20 \mu \mathrm{W}$, achieved only under certain processing conditions (Wunderlich 2009) similar to the reduced atmosphere required for Nb-doped $\mathrm{SrTiO}_{3}$ (Ohta et al. 2005). New results showed that impurities of $\mathrm{C}, \mathrm{V}$, and Co deteriorate the Seebeck coefficient, and other co-doping studies are in progress. $\mathrm{Zn}$ - and Co-oxide ceramics are easy to sinter, but we have not yet found a composition with sufficiently high Seebeck coefficient or current (Wunderlich 2013a). Silicides are also difficult to process and require SPS (Wunderlich et al. 2014b), because the high $\mathrm{Mg}$ vapor pressure prevents conventional arc-melting. Another such difficult to process element is $\mathrm{Sb}$, due to its high vapor pressure. Similarly, $\mathrm{Cr}$, and Mo are difficult, because they possess slow diffusion coefficients and high melting points. Pure $\mathrm{Zr}, \mathrm{Ba}$, and other rare earth elements are delivered in oil, because they easily oxidize making their handling difficult. We could not avoid that specimens contained traces of oil, which caused pores in the arc-melted specimens. Nevertheless, the BaCuSi clathrates can be produced by conventional arc-melting and seem to be less sensitive to deviations from stoichiometry; only traces of $\mathrm{O}$ and to a less extent $\mathrm{C}$, deteriorate the Seebeck coefficient. This is the crucial point in processing of any intermetallics. Surface oxidation starts above $600^{\circ} \mathrm{C}$ in $\mathrm{BaCuSi}$, (Ti,Zr)NiSn, $\mathrm{Mg}_{2} \mathrm{Si}$ and the contacting $\mathrm{Ni}$ wires, but have not been found to have large influence on the Seebeck voltage.

Hence, the result of this overall judgment is that (TiZr)NiSn specimens show performance with cheapest manufacturing expenses. Their thin films are $1 \mu \mathrm{m}$ thickness; however, they have not yet reached the performance of bulk specimens, mainly because the sputter ratios of the four elements $\mathrm{Ti}, \mathrm{Zr}, \mathrm{Ni}, \mathrm{Sn}$ are so different. On the other hand, we could confirm another finding that $\mathrm{Cu}$ substrates are beneficial compared to others (Wunderlich 2014c), because the carrier concentration is enlarged. Thus, TE integration into microchips is possible and will have a large impact in the field of TE and functional metals in the near future (Wunderlich 2014c).

\section{Conclusion}

We report here for the first time a four-step measuring method for characterizing TEs. This method is based on the closing of the electric circuit with load resistors and the resulting change in carrier concentrations. The results are summarized as:

1) As seen in the $I-U$ plot, the output power of $0.01 \mathrm{~mW}$ at $\Delta T=650 \mathrm{~K}$ was achieved for most tested specimens, which corresponds to the reported $Z T$ values of 0.4 .

2) In the plot of output power as a function of load resistance metallic-like specimens show highest power in the region of high carrier concentration, while ceramic-like specimens get exhausted above a certain concentration, which strongly depends on the temperature.

3) In the power-versus- temperature plot metallic-like specimens have almost the same activation energies for all carrier concentrations and their low internal resistance can be confirmed by circuit theory analysis. Ceramic-like specimens show a strong dependence on carrier concentration with different activation energies. The isosbestic-like intersection points can explain the maximum reachable power output, which is explained by their large effective mass. 


\section{References}

Bulusu, A., and D. G. Walker. 2008. "Review of Electronic Transport Models for Thermoelectric Materials." Superlattices and Microstructures 44:1-36. doi:10.1016/j.spmi.2008.02.008.

Clerc, J. P., G. Giraud, J. M. Laugier, and J. M. Luck. 1990. "The Electrical Conductivity of Binary Disordered Systems, Percolation Clusters, Fractals and Related Models." Advances in Physics 39:191. doi:10.1080/0001873900010150.

Culp, S. R., S. J. Poon, T. M. Tritt, et al. 2006. "Effect of Substitutions on the Thermoelectric Figure of Merit of Half-Heusler Phases at $800{ }^{\circ} \mathrm{C} . "$ Applied Physics Letters 88:042106. doi:10.1063/ 1.2168019.

De Marchi, A., and V. Giaretto. 2011. "An Accurate New Method to Measure the Dimensionless Figure of Merit of Thermoelectric Devices Based on the Complex Impedance Porcupine Diagram." Review of Scientific Instruments 82:104904. doi:10.1063/ 1.3656074

Feldhoff, A., and B. Geppert. 2014. "A High-Temperature Thermoelectric Generator Based on Oxides." Energy Harvesting and Systems 1 (1-2):69-78. doi:10.1515/ehs-2014-0003.

Gelbstein, Y., J. Tunbridge, R. Dixon, M. J. Reece, H. Ning, R. Gilchrist, R. Summers, I. Agote, M. A. Lagos, K. Simpson, et al. 2014. "Physical, Mechanical, and Structural Properties of Highly Efficient Nanostructured n- and p-Silicides for Practical Thermoelectric Applications." Journal of Electronic Materials 43:1703-11. doi:10.1007/s11664-013-2848-9.

Goldschmid, H. J., and G. Douglas. 1954. "The Use of Semiconductors Refrigerators." British Journal of Applied Physics 5 (4):386-90.

Goupil, C., W. Seifert, K. Zabrocki, E. Müller, and G. Snyder. 2011. "Thermodynamics of Thermoelectric Phenomena and Applications." Entropy 13:1481-517. doi:10.3390/e13081481.

Liu, W., Q. Zhang, K. Yin, H. Chi, X. Zhou, X. Tang, and C. Uher. "High Figure of Merit and Thermoelectric Properties of Bi-Doped $\mathrm{Mg}_{2} \mathrm{Si}_{0.4} \mathrm{Sn}_{0.6}$ Solid Solutions." Journal of Solid State Chemistry 2013 203:333-9. doi:10.1016/j.jssc.2013.04.041.

Min, G. 2010. "ZT Measurements under Large Temperature Differences." Journal of Electronic Materials 39:1782-5. doi:10.1007/s11664-010-1136-1.

Muta, H., T. Kanemitsu, K. Kurosaki, and S. Yamanaka. 2006. "Substitution Effect on Thermoelectric Properties of ZrNiSn Based Half-Heusler Compounds." Materials Transactions 47 (6):1453-7.

Muto, A., D. Kraemer, Q. Hao, Z. F. Ren, and G. Chen. 2009. "Thermoelectric Properties and Efficiency Measurements Under Large Temperature Differences." Review of Scientific Instruments 80:093901. doi:10.1063/1.3212668.

Ohta, H., et al. 2007. "Giant Thermoelectric Seebeck Coefficient of a Two-Dimensional Electron Gas in $\mathrm{SrTiO}_{3}$." Nature Materials 6:129-34. doi:10.1038/nmat1821.

Ohta, S., T. Nomura, H. Ohta, and K. Koumoto. 2005. “HighTemperature Carrier Transport and Thermoelectric Properties of Heavily La- or Nb-Doped $\mathrm{SrTiO}_{3}$ Single Crystals." Journal of Applied Physics 97:034106. doi:10.1063/1.1847723.
Paschen, S., W. Carrillo-Cabrera, A. Bentien, V. H. Tran, M. Baenitz, Y. Grin, and F. Steglich. 2001. "Structural, Transport, Magnetic, and Thermal Properties of $\mathrm{Eu}_{8} \mathrm{Ga}_{16} \mathrm{Ge}_{30}$." Physical Review $B$ 64:214404. doi:10.1103/PhysRevB.64.214404.

Sakurada, S., and N. Shutoh. 2005. "Effect of Ti Substitution on the Thermoelectric Properties of ( $\mathrm{Zr}, \mathrm{Hf}) \mathrm{NiSN}$ Half-Heusler Compounds." Applied Physics Letters 86:082105. doi:10.1063/ 1.1868063.

Sandoz-Rosado, E. J., S. J. Weinstein, and R. J. Stevens. 2013. "On the Thomson Effect in Thermoelectric Power Devices." International Journal of Thermal Sciences 66:1-7. doi:10.1016/ j.ijthermalsci.2012.10.018.

Sootsman, J. R., D. Y. Chung, and M. G. Kanatzidis. 2009. Angewandte Chemie 121:8768-92. 10.1002/ange.200900598.

Vining, C. B. 1991. "A Model for the High-Temperature Transport Properties of Heavily Doped n-Type Silicon-Germanium Alloys." Journal of Applied Physics 69:331. doi:10.1063/1.347717.

Wang, C. -Y., R. Pagel, J. K. Dohrmann, and D. W. Bahnemann. 2006. "Antenna Mechanism and Deaggregation Concept: Novel Mechanistic Principles for Photocatalysis." Comptes Rendus Chimie 9:761-73.

Whitney, R. S. 2014. "Most Efficient Quantum Thermoelectric at Finite Power Output.” Physical Review Letters 112:130601. doi:10.1103/PhysRevLett.112.130601.

Wunderlich, W. 2009. " $\mathrm{NaTaO}_{3}$ Composite Ceramics - A New Thermoelectric Material for Energy Generation." Journal of Nuclear Materials 389:57-61. doi:10.1016/j.jnucmat.2009.01.007 (online 13.01.2009).

Wunderlich, W. 2013a "Large Seebeck Voltage of Co, Mn, Ni, FeCeramics." Advances in Ceramic Science and Engineering (ACSE) 2:9-15. http://www.acse-journal.org/Download.aspx? $\mathrm{ID}=5579$.

Wunderlich, W. 2014c. "The Atomistic Structure of Metal/Ceramic Interfaces Is the Key Issue for Developing Better Properties." Metals 4:410-27. doi:10.3390/met4030410.

Wunderlich, W., M. Amano, and Y. Matsumura. 2014a. "Electronic Band-Structure Calculations of $\mathrm{Ba}_{8} \mathrm{Me}_{x} \mathrm{Si}_{46-x}$ Clathrates with $\mathrm{Me}=\mathrm{Mg}, \mathrm{Pd}, \mathrm{Ni}, \mathrm{Au}, \mathrm{Ag}, \mathrm{Cu}, \mathrm{Zn}, \mathrm{Al}, \mathrm{Sn}$.” Journal of Electronic Materials 43:1527-32. doi:10.1007/s11664-013-2770-1.

Wunderlich, W., and Y. Motoyama. 2009. "Screening and Fabrication of Half-Heusler Phases for Thermoelectric Applications." Materials Research Society Symposium Proceedings 1128-U0110:1-6. doi:10.1557/PROC- 1128-U01-10.

Wunderlich, W., and H. Ohsato. 2013b. "Enhanced Microwave Resonance Properties by Structural Analysis of the PseudoTungstenbronze $\mathrm{Ba}_{6-3 \times} \mathrm{R}_{8+2 x} \mathrm{Ti}_{18} \mathrm{O}_{54}(\mathrm{R}=$ Rare Earth) Solid Solutions." Japanese Journal of Applied Physics 52:09KH04. doi:10.7567/JJAP.52.09KH04.

Wunderlich, W., Y. Suzuki, N. Gibo, T. Ohkuma, M. Al-Abandi, M. Sato, A. U. Khan, and T. Mori. 2014b "Thermoelectric Properties of $\mathrm{Mg}_{2} \mathrm{Si}$ Produced by New Chemical Route and SPS." Inorganics 2:351-62. doi:10.3390/inorganics2020351.

Yan, X., M. X. Chen, S. Laumann, E. Bauer, P. Rogl, R. Podloucky, and S. Paschen. 2012. "Thermoelectric Properties of Ba-Cu-Si Clathrates." Physical Review B 85:165127. doi:10.1103/ PhysRevB.85.165127. 